\title{
Studying the Stability of Some Models Combinatory with Application
}

\author{
Abdulghafoor Salim \\ drabdul_salim@uomosul.edu.iq \\ College of Computer Science and \\ Mathematics, University of Mosul,
}

\author{
Raad Awad Alhamdani \\ College of Education \\ University of Tikrit,
}

Received on: 17/5/2010

Accepted on: 16/8/2010

\begin{abstract}
In this paper we find the statistical properties (Moments) of the harmonic model with additive noise, The stability of the mixed spectra (linear and non-linear models) for special case (low order) by using the Ozaki linear approximation method is found .The time series of the mean monthly temperature of Bege City is applied in order to explain the studied method. A mathematical model SARIMA $(1,0,0)(2,0,0)$ s is suggested by the NBIC criterion and other statistical tools (auto-correlation and the residual variance). A one year ahead prediction is made for the studied time series by using the proposed model .

Key word : Stationarity, Harmonic Model, Mixed Spectra Model, Limit Cycle

$$
\text { دراسة استقرارية بعض النماذج التوافقية مع تطبيق }
$$

$$
\text { كلد عواد الحمداني التربية }
$$

تاريخ قبول البحث: 2010/8/16

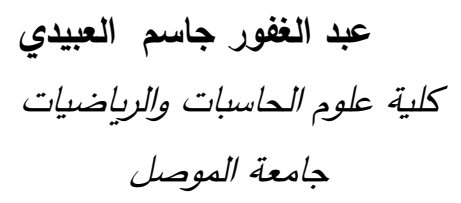

\section{الملخص}

يتم في هذه البحث إيجاد الصفات الإحصائية (العزوم) للنموذج التوافقي مضافا له حد الخطأ العشوائي

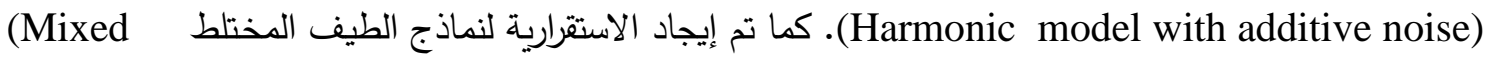
spectra Models)

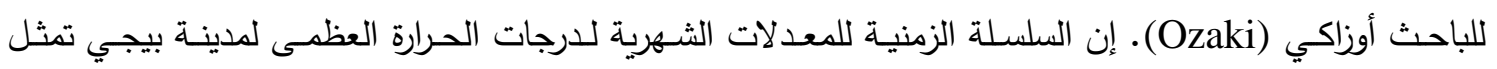
الجانب التطبيقي في دراستتا حيث يتم بناء نماذج رياضية مختلفة لإيضاح الطريقة التي تمت دراستها وتم اقتراح أنموذج SARIMA(1,0,0)(2,0,0)s ليمثل السلسلة الزمنية المدروسة وذلك باستخدام معيار NBIC الوسائل الإحصائية الأخرى (الارتباط الذاتي وتباين البواقي). وقد تم التتبؤ بالقيم المستقبلية لسنة واحدة باستخدام الأنموذج المقترح. الكلمات المفتاحية: ثابت ، نموذج متتاسق ، مختلط الأطياف ، دورة الحد
\end{abstract}


سنركز دراستتا في هذا البحث على إيجاد العزوم للنموذج التوافقي وكذلك دراسة استقرارية نموذج الطيف

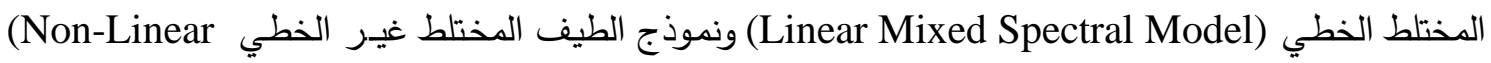
Mixed Spectral Model)

2-2 المقدمة Introduction

تبنى نماذج السلاسل الزمنية عادةً على خصائص أساسية متمثلة بالاستقرارية (Stationarity) والخطية

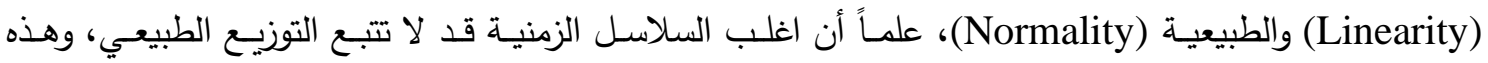

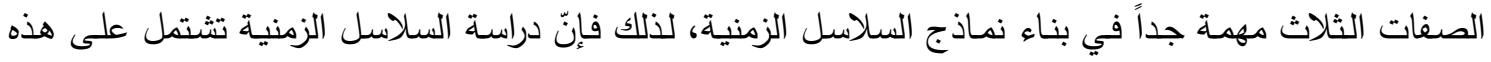

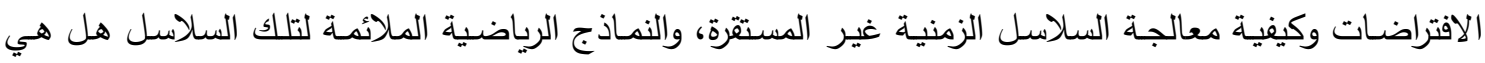
خطية أم غير خطية وفيما إذا كانت تتبع التوزيع الطبيعي أم لا، وسنحاول عرض بعضه وضل الدراسات في هذا المجال

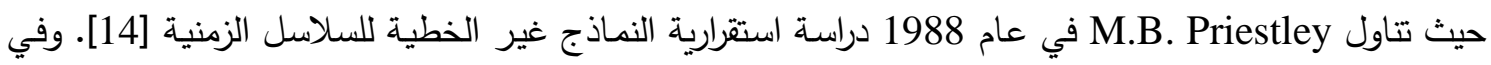
عام (1999) قام كلّ من (Kurt Hornik, Fredrick, I) بدراسة الاستقرارية في الانحدار الذاتي للشبكات) [9].

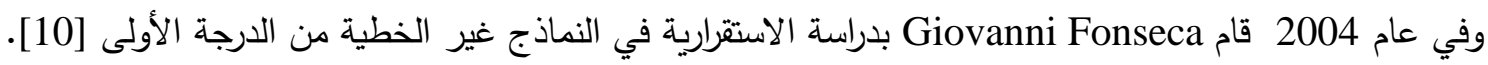
في العام (2005) قام الباحث أزهر عباس الحيالي بدراسـة استقرارية النموذج اللوجستي (2] LSTAR(p). وسنحاول في هذا البحث دراسة الاستقرارية لبعض النماذج التي تمتلك صفات دورية وباستخدام طريقة التقريب الخطي للباحث الياباني أوزاكي.

\section{3- الجانب النظري ومفاهيم أساسية:}

سوف نستعرض بعض المفاهيم والتعاريف الأساسية المهمة التي نحتاجها في الجانب النظري:

[8] [7] Stationarity الاستقرارية 1-3

تكون السلسلة الزمنية \}

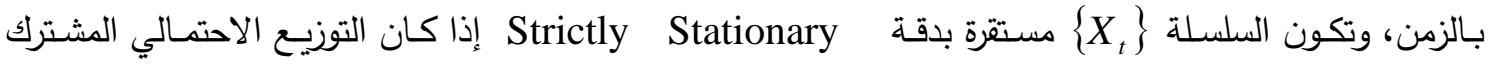
للمتغيرات $X_{t_{1}}, X_{t_{2}}, \ldots . ., X_{t_{n}}$ هو نفس التوزيع الاحتمالي المشترك للمتغيرات $X_{t_{1+k}}, X_{t_{2+k}}, \ldots . ., X_{t_{n+k}}$

ولجميع النقاط الزمنية المختارة

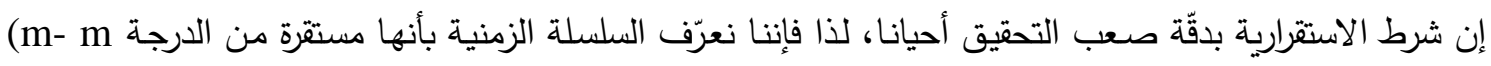

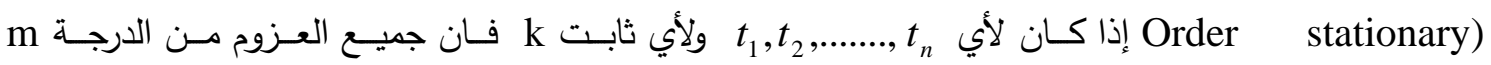
.[13] تكون $\left(X_{t_{1+k}}, X_{t_{2+k}}, \ldots . ., X_{t_{n+k}}\right)$ $E\left[\left\{X_{t_{1}}\right\}^{m_{1}},\left\{X_{t_{2}}\right\}^{m_{2}}, \ldots \ldots \ldots \ldots,\left\{X_{t_{n}}\right\}^{m_{n}}\right]=E\left[\left\{X_{t_{1+k}}\right\}^{m_{1}},\left\{X_{t_{2+k}}\right\}^{m_{2}}, \ldots \ldots \ldots \ldots,\left\{X_{t_{n+k}}\right\}^{m_{n}}\right]$

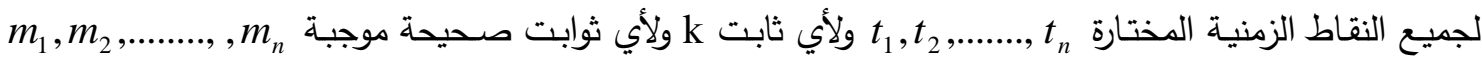
تحقق القيد $m_{1}+m_{2}+\ldots \ldots . .+m_{n} \leq m$ 
ولحالة خاصة يقال إن السلسلة الزمنية $\left\{X_{t}\right.$ أنها مستقرة من الرتبة الأولى First Order stationary) إذا كانت: $E\left(X_{t}\right)=\mu$

كما يقال للسلسلة الـزمنية $\left\{X_{t}\right.$ أنها مستقرة من الـتبة الثانية (secondary Order stationary) إذا حققت

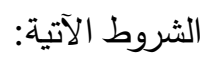

1) $E\left(X_{t}\right)=\mu$

2) $\operatorname{Var}\left(X_{t}\right)=\sigma_{x}^{2}$

t كمية ثابتة لا تعتمد على

3) $\operatorname{Cov}\left[X_{t_{1}}, X_{t_{2}}\right]=\gamma_{\left(t_{1}, t_{2}\right)}$

t $\sigma_{x}^{2}$

دالة بدلالة | $\mid t_{2}-t_{1}$

[6] Harmonic With Additive White Noise 2-3 النموذج التوافقي مضافاً له حد الخطأ نمـوذج الانحـدار الذاتي التوافقي وللاختصـار (النموذج التوافقي) يتضــن الجيوب وجيوب التمـام مـع التكرارات

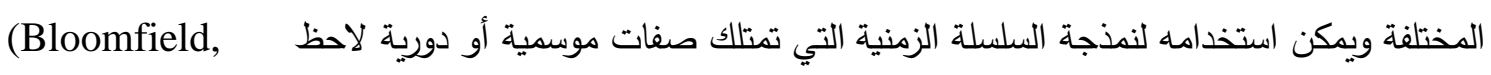
(1976 يقال للسلسلة الزمنية بأنهّا تحقق النموذج التوافقي بـ (m) من المركبات إذا حققت المعادلة الآتية: $X_{t}=\sum_{j=1}^{m} \alpha_{j} \cos \left(2 \pi w_{j} t+\phi_{j}\right)+Z_{t}$

إذ

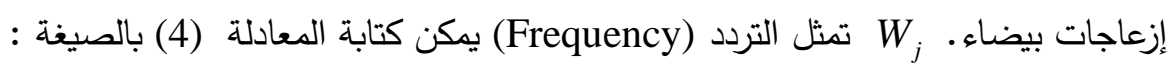
$X_{t}=\sum_{j=1}^{m} A_{j} \cos \left(w_{j} t\right)+B_{j} \sin \left(w_{j} t\right)+Z_{t}$

[6] (Bloomfield) لاحظ

$$
\begin{gathered}
\alpha_{j}=\sqrt{A_{j}^{2}+B_{j}^{2}} \\
A_{j}=\alpha_{j} \cos \left(\phi_{j}\right) \\
B_{j}=-\alpha_{j} \sin \left(\phi_{j}\right)
\end{gathered}
$$

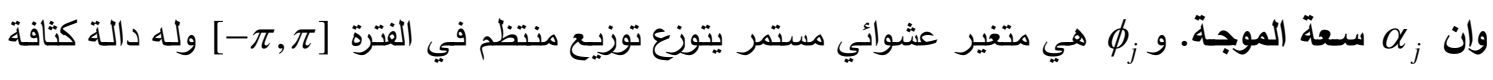
$f\left(\phi_{j}\right)=\frac{1}{2 \pi}, \quad-\pi \leq \phi_{j} \leq \pi$ 
Mixed Spectral Model (Linear) 3-3 نموذج الطيف المختلط الخطي يتكون نموذج الطيف المختلط الخطي من نموذج توافقي مضاف إليه نموذج انحدار ذاتي مع الإزعاجات البيضـاء

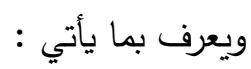
$X_{t}=\sum_{j=1}^{m} \alpha_{j} \cos \left(2 \pi w_{j} t+\phi_{j}\right)+\sum_{j=1}^{n} \beta_{j} X_{t-j}+Z_{t}$

حيث

3-4 نموذج الاتحدار الذاتي الأسي (12] Exponential Autoregressive Model (EXPAR (p) عرّف الباحث Ozaki عام 1985 نموذج الانحدار الذاتي الأسي من الرتبة p بالثكل الأتي: $X_{t}=\sum_{i=1}^{p}\left(\alpha_{i}+\pi_{i} e^{-x_{t-1}^{2}}\right) X_{t-i}+Z_{t}$

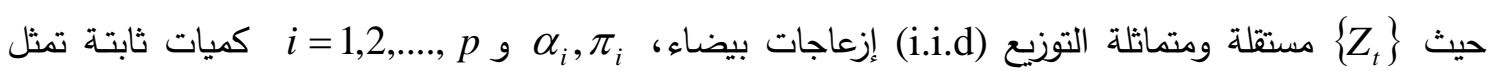
معلمات (parameters) النموذج.

Mixed Spectral Model (Non-Linear) 5موذج الطيف المختلط غير الخطي يمكن تعريف نموذج الطيف المختلط غير الخطي على انهُ مكون من حدين. الحد الأول النموذج التوافقي والحد

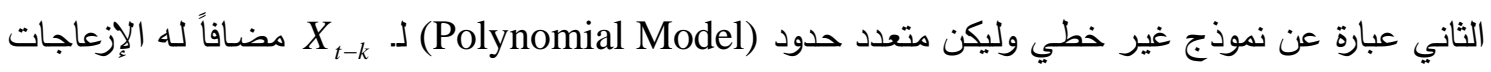
البيضاء أي أنّ: $X_{t}=\sum_{j=1}^{m} \alpha_{j} \cos \left(2 \pi w_{j} t+\phi_{j}\right)+\sum_{j=1}^{p} \beta_{j} X_{t-j}^{p}+Z_{t}$

حيث p عدد صحيح و و 6-3 أنموج الاحدار الذاتي والأوساط المتحركة المندمج [1] (Autoregressive Integrated Moving Average Model) ARIMA(p,d,q)

نظراً لان اغلب السلاسل الزمنية المستعملة في المجالات التطبيقية غير مستقرة فلابد من تحويلها إلى سلاسل

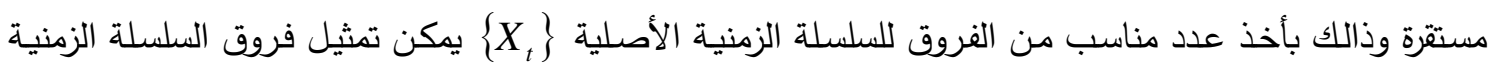
$\nabla^{d} X_{t}=(1-B)^{d} X_{t}$

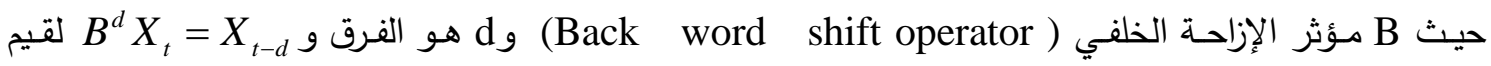
. $d=1,2,3, \ldots$.

وبعد اخذ عدد مناسب من الفروق وليكن d للسلسلة الأصلية

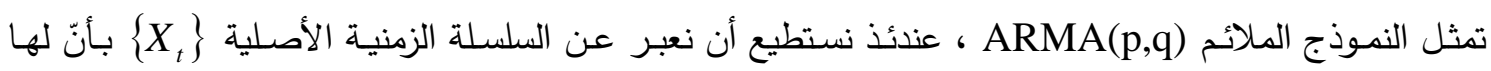

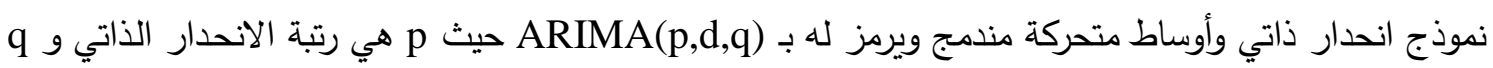
$\phi(B) \nabla^{d} X_{t}=\theta(B) Z$ هي رتبة الأوساط المتحركة وd هو الفرق. ويعبر عن النموذج بالثكل الآتي: 
$\phi_{p}(B)=1-\phi_{1} B-\phi_{2} B^{2}-\ldots \ldots . . \phi_{p} B^{p}$

$\theta_{q}(B)=1-\theta_{1} B-\theta_{2} B^{2}-\ldots \ldots \ldots-\theta_{q} B^{q} \quad$,

وان

(1) $E\left(z_{t}\right)=0$

(2) $\operatorname{Var}\left(z_{t}, z_{t \pm k}\right)=\left\{\begin{array}{l}\sigma^{2} z ; k=0 \\ 0 \quad ; k \neq 0\end{array}\right.$

(3) $\operatorname{Cov}\left(\mathrm{z}_{t}, z_{t \pm k}\right)=0, \forall k \neq 0$

[15][11] Fixed Point تعريف (النقطة المنفردة) 7-3

تعرف النقطة المنفردة $\xi$ للنموذج

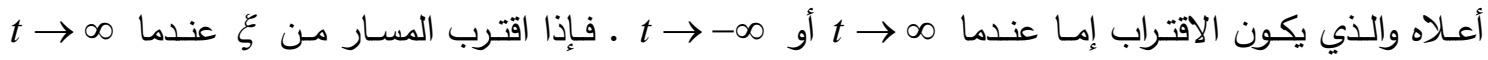

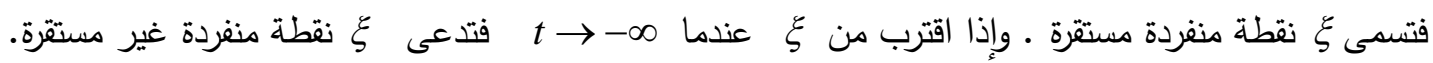

[15] [11] Limit cycle عريف (دورة النهاية) 8-3

$X_{t}=f\left(x_{t-1}, \ldots ., x_{t-p}\right)$

ليكن لدينا النموذج الأتي

تعرف دورة النهايـة للنموذج أعـلاه على أنها المسـار المعزول والمغلق

صحيح موجب تمثل الدورة.

ويعرف المسار المعزول بأنة ذالك المسار الذي يقترب جداً من دورة النهاية، إما المسار المغلق فهو قيم ابتدائية

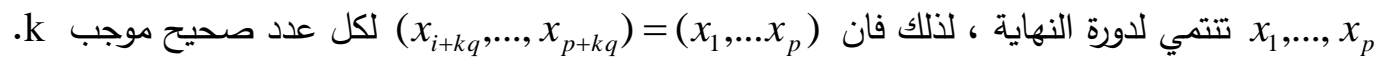

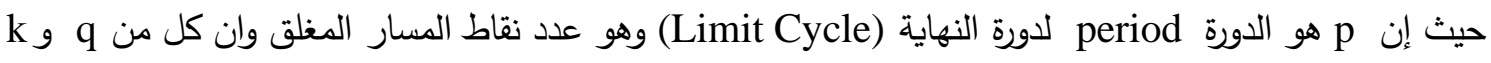
عدد صحيح موجب.

9-3 النموذج التوافقي مضافاً له حد الخطأ:

يمتلك النموذج التوافقي صفات دوريـة وكما لاحظنا من خـلال التعريف فهو مكون من مركبتين دوريتين (الجيب

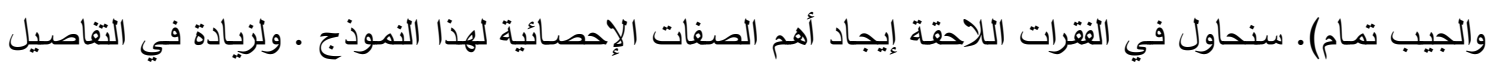

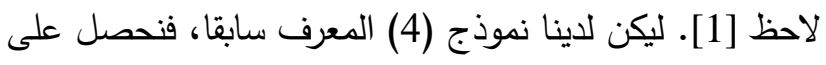

$$
\begin{aligned}
& \text { 1- القيمة المتوقعة: } 0\left(X_{t}\right)=0 \\
& \gamma_{0}=\sum_{j=1}^{m} \frac{\alpha_{j}^{2}}{2}+\sigma_{z}^{2}: \text {-2 } \\
& \gamma_{r}=\sum_{j=1}^{m} \frac{\alpha_{j}^{2}}{2}\left[\cos \left(w_{j} r\right)\right] \text { : دالّة التغاير }
\end{aligned}
$$


$\rho_{r}=\frac{\sum_{j=1}^{m} \alpha_{j}^{2}\left[\cos \left(w_{j} r\right)\right]}{\sum_{j=1}^{m} \alpha_{j}^{2}+2 \sigma_{z}^{2}}, \quad r=1,2, \ldots, m$

بما إن العزوم للنموذج التوافقي لا تعتمد على t فهذا يشير إلى أن 10-3 نموذج الطيف المختلط الخطي:

سوف نحاول إيجاد الصفات الإحصائية لحالة خاصة من نموذج الطيف المختلط الخطي. أي عندما = ز $X_{t}=\alpha \cos (2 \pi w t+\phi)+\beta X_{t-1}+Z_{t}$

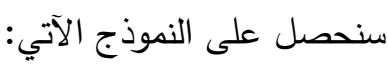

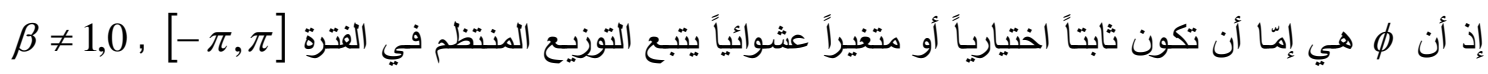
و 0 \& ، ويمكن إيجاد الصفات الإحصائية لنموذج الطيف المختلط الخطي العام بالطريقة نفسها.

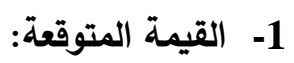
أ - إذا كانت و كمية ثابتة فان

$$
\begin{aligned}
& E\left(X_{t}\right)=E(\alpha \cos (2 \pi w t+\phi))+E\left(\beta X_{t-1}\right)+E\left(Z_{t}\right) \\
& E\left(X_{t}\right)=\alpha \cos (2 \pi w t+\phi)+\beta E\left(X_{t-1}\right)+0 \\
& \mu_{x}=\alpha \cos (2 \pi w t+\phi)+\beta \mu_{x}+0 \\
& \mu_{x}(1-\beta)=\alpha \cos (2 \pi w t+\phi) \\
& E\left(X_{t}\right)=\mu_{x}=\frac{\alpha}{(1-\beta)} \cos (2 \pi w t+\phi)
\end{aligned}
$$

حيث نلاحظ أنّ القيمة المتوقعة لـ

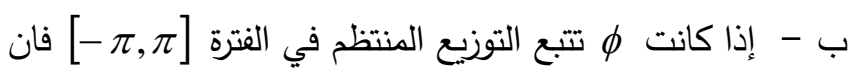

$$
\begin{aligned}
& E\left(X_{t}\right)=E(\alpha \cos (2 \pi w t+\phi))+E\left(\beta X_{t-1}\right)+0 \\
& =\alpha(\cos (2 \pi w t) E[\cos (\phi)]-\sin (2 \pi w t) E[\sin (\phi)])
\end{aligned}
$$

$$
E(\cos (\phi))=E(\sin (\phi))=0
$$

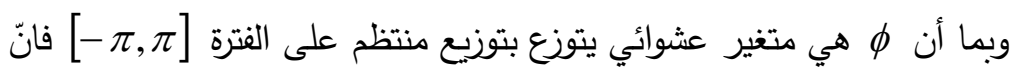

$$
\begin{aligned}
E(\cos (\phi)) & =\int_{-\pi}^{\pi}(\cos (\phi)) f(\phi) d \phi \\
& =\frac{1}{2 \pi} \int_{-\pi}^{\pi} \cos (\phi) d \phi=\left.\frac{1}{2 \pi} \sin (\phi)\right|_{-\pi} ^{\pi}
\end{aligned}
$$




$$
\begin{aligned}
& =\frac{1}{2 \pi}[\sin (\pi)-\sin (-\pi)] \\
& =\frac{1}{2 \pi}[0+0]=0
\end{aligned}
$$

كذلك

$$
\begin{aligned}
E(\sin (\phi)) & =\int_{-\pi}^{\pi}(\sin (\phi)) f(\phi) d \phi \\
& =\frac{1}{2 \pi} \int_{-\pi}^{\pi} \sin (\phi) d \phi=\frac{1}{2 \pi}\left[-\left.\cos (\phi)\right|_{-\pi} ^{\pi}\right] \\
& =\frac{1}{2 \pi}[-\cos (\pi)+\cos (-\pi)] \\
& =\frac{1}{2 \pi}[-1+1]=\frac{1}{2 \pi}[0]=0
\end{aligned}
$$$$
E(\cos (2 \pi w t+\phi))=0
$$$$
\text { نعوض عن قيمة كل من [ [e }
$$

نعوض عن قيمة المعادلة (13) في المعادلة (11) نحصل على

$$
\begin{aligned}
& E\left(X_{t}\right)=\alpha(0)+\beta E\left(X_{t-1}\right) \\
& E\left(X_{t}\right)=\beta E\left(X_{t-1}\right) \\
& \mu=\beta \mu \\
& (1-\beta) \mu=0 \\
& E\left(X_{t}\right)=0
\end{aligned}
$$$$
\text { بما أنّ 1,0 }
$$

$$
\operatorname{var}\left(X_{t}\right)=E\left(X_{t}^{2}\right)-\left[E\left(X_{t}\right)\right]^{2}
$$

$$
\begin{aligned}
& \operatorname{var}\left(X_{t}\right)=E\left(X_{t}^{2}\right)-0=E\left(X_{t}^{2}\right) \\
& \begin{aligned}
X_{t}=\alpha(\cos (2 \pi w t+\phi))+\beta X_{t-1}+Z_{t} \\
X_{t}^{2}=\left[\alpha(\cos (2 \pi w t+\phi))+\beta X_{t-1}+Z_{t}\right]^{2} \\
=[\alpha(\cos (2 \pi w t+\phi))]^{2}+\beta^{2} X_{t-i}^{2}+Z_{t}^{2}+ \\
\quad 2 \alpha \beta(\cos (2 \pi w t+\phi)) X_{t-1}+2 \alpha(\cos (2 \pi w t+\phi)) Z_{t}+2 \beta X_{t-1} Z_{t}
\end{aligned}
\end{aligned}
$$

وبأخذ القيمة المتوقعة للطرفين نحصل على $\left.E\left(X_{t}^{2}\right)=\alpha^{2} E[(\cos (2 \pi w t+\phi))]^{2}+E\left(\beta^{2} X_{t-i}^{2}\right)+E Z_{t}^{2}\right)+$

$$
2 \alpha \beta E\left[(\cos (2 \pi w t+\phi)) X_{t-1}\right]+2 \alpha E\left[(\cos (2 \pi w t+\phi)) Z_{t}\right]+2 \beta E\left[X_{t-1} Z_{t}\right]
$$

$\left\{Z_{t}\right\}$ بما أن $E\left[X_{t}^{2}\right]=\alpha^{2} E\left[\left(\cos ^{2}(2 \pi w t+\phi)\right)\right]+\beta^{2} E\left[X_{t-i}^{2}\right]+\sigma_{z}^{2}+0+0+0$ 


$$
\begin{aligned}
\gamma_{0}=\alpha^{2} E\left[\left(\cos ^{2}(2 \pi w t\right.\right. & +\phi))]+\beta^{2} \gamma_{0}+\sigma_{z}^{2} \\
E\left[\left(\cos ^{2}(2 \pi w t+\phi)\right)\right] & =E\left\{\frac{1}{2}[1+(\cos (4 \pi w t+2 \phi))]\right\} \\
& =\frac{1}{2}+\frac{1}{2} E[(\cos (4 \pi w t+2 \phi))] \\
& =\frac{1}{2}+\frac{1}{2} E[\cos (4 \pi w t) \cos (2 \phi)-\sin (4 \pi w t) \sin (2 \phi)] \\
& =\frac{1}{2}+\frac{1}{2}[\cos (4 \pi w t) E(\cos (2 \phi))-\sin (4 \pi w t) E(\sin (2 \phi))]
\end{aligned}
$$

$$
\begin{aligned}
E(\cos (2 \phi)) & =\int_{-\pi}^{\pi}(\cos (2 \phi)) f(\phi) d \phi \\
& =\frac{1}{2 \pi} \int_{-\pi}^{\pi} \cos (2 \phi) d \phi=\left.\frac{1}{4 \pi} \sin (2 \phi)\right|_{-\pi} ^{\pi} \\
& =\frac{1}{4 \pi}[\sin (2 \pi)-\sin (-2 \pi)] \\
& =\frac{1}{4 \pi}[0+0],=0 \\
E(\sin (2 \phi)) & =\int_{-\pi}^{\pi}(\sin (2 \phi)) f(\phi) d \phi \\
& =\frac{1}{2 \pi} \int_{-\pi}^{\pi} \sin (2 \phi) d \phi=\frac{1}{4 \pi}\left[-\left.\cos (2 \phi)\right|_{-\pi} ^{\pi}\right] \\
& =\frac{1}{4 \pi}[-\cos (2 \pi)+\cos (-2 \pi)] \\
& =\frac{1}{4 \pi}[-1+1] \\
& =\frac{1}{4 \pi}[0]=0
\end{aligned}
$$

$E\left(\cos ^{2}(2 \pi w t+\phi)\right)=\frac{1}{2}+0+0=\frac{1}{2}$

نعوّض عن قيمة (15) في المعادلة (14) فنحصل على أنّ

$$
\begin{aligned}
& \gamma_{0}=\frac{\alpha^{2}}{2}+\beta^{2} \gamma_{0}+\sigma_{z}^{2} \\
& \gamma_{0}-\beta^{2} \gamma_{0}=\frac{\alpha^{2}}{2}+\sigma_{z}^{2} \\
& \left(1-\beta^{2}\right) \gamma_{0}=\frac{\alpha^{2}}{2}+\sigma_{z}^{2} \\
& \gamma_{0}=\frac{\alpha^{2}}{2\left(1-\beta^{2}\right)}+\frac{2 \sigma_{z}^{2}}{2\left(1-\beta^{2}\right)}=\frac{\alpha^{2}+2 \sigma_{z}^{2}}{2\left(1-\beta^{2}\right)}
\end{aligned}
$$




$$
\gamma_{0}=\frac{\alpha^{2}+2 \sigma_{z}^{2}}{2\left(1-\beta^{2}\right)}
$$

$$
\gamma_{k}=\operatorname{cov}\left(X_{t}, X_{t+k}\right)=E\left(X_{t} X_{t+k}\right)-E\left(X_{t}\right) E\left(X_{t+k}\right)
$$

$$
\left.\gamma_{k}=\operatorname{cov}\left(X_{t}, X_{t+k}\right)=E\left(X_{t} X_{t+k}\right)\right)
$$

$E\left(X_{t} X_{t+k}\right)=\alpha^{2} E[(\cos (2 \pi w t+\phi))(\cos (2 \pi w(t+k)+\phi))]$

$$
+\alpha \beta E\left[(\cos (2 \pi w(t+k)+\phi)) X_{t-1}\right]+\beta^{2} E\left[X_{t-1} X_{t+k-1}\right]
$$

$\gamma_{k}=\alpha^{2}\left(\cos (2 \pi w k) E\left[\left(\cos ^{2}(2 \pi w t+\phi)\right]-\alpha^{2} \sin (2 \pi w k)\right.\right.$

$$
E[\sin (2 \pi w t+\phi) \cos (2 \pi w t+\phi)]+\alpha \beta\left(\operatorname { c o s } ( 2 \pi w k ) E \left[X_{t-1}(\cos (2 \pi w t+\phi)]\right.\right.
$$$$
-\alpha \beta \sin (2 \pi w k) E\left[\sin (2 \pi w t+\phi) X_{t-1}\right]+\beta^{2} \gamma_{k}
$$

$\gamma_{k}-\beta^{2} \gamma_{k}=\frac{\alpha^{2}(\cos (2 \pi w k)}{2}-\alpha^{2} \sin (2 \pi w k)(0-0)+\alpha \beta(\cos (2 \pi w k)(0-0)$

$\left(1-\beta^{2}\right) \gamma_{k}=\frac{\alpha^{2}(\cos (2 \pi w k)}{2}$

$\gamma_{k}=\frac{\alpha^{2}(\cos (2 \pi w k)}{2\left(1-\beta^{2}\right)}$

$\rho_{k}=\frac{\gamma_{k}}{\gamma_{0}}=\frac{\frac{\alpha^{2}(\cos (2 \pi w k)}{2\left(1-\beta^{2}\right)}}{\frac{\alpha^{2}+2 \sigma_{z}^{2}}{2\left(1-\beta^{2}\right)}}$

$\rho_{k}=\frac{\alpha^{2}(\cos (2 \pi w k)}{\alpha^{2}+2 \sigma_{z}^{2}}$

نلاحظ أن الصفات الإحصائية لهذا النموذج لا تعتمد على t وهذا ما يؤدي إلى أنّ نموذج الطيف المختط الخطي يكون مستقراً.

نستتج من ذلك انه إذا كانت و كمية ثابتة فان نموذج الطيف المختلط الخطي يكون غير مستقراً إذ نلاحظ أنّ النّا الصفات الإحصائية تعتمد على t. وإذا كانت ه بتتع التوزيع المنتظم يكون نموذج الطيف المختلط الخطي مستقراً. 11-3 استقرارية نموذج الطيف المختلط غير الخطي:

سوف نحاول إيجاد الاستقرارية لحالة خاصة من نموذج الطيف المختلط غير الخطي أي عندما p=1,2 في (8). $X_{t}=\alpha \cos (2 \pi w t+\phi)+\beta_{1} X_{t-1}+\beta_{2} X_{t-1}^{2}+Z_{t}$

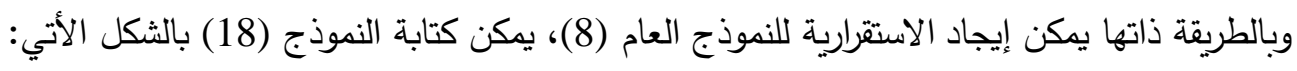
$X_{t}=\alpha \cos (2 \pi w t+\phi)+H_{t}$

$$
H_{t}=\beta_{1} X_{t-1}+\beta_{2} X_{t-1}^{2}+Z_{t}
$$


إذ من الدرجة الثانية سنحاول إيجاد استقرارية النموذج (20) باستخدام طريقة التقريب لأوزاكي وكما يأتي. ليكن لدينا النموذج $X_{t}=\beta_{1} X_{t-1}+\beta_{2} X_{t-1}^{2}+Z_{t}$

1- نجد النقطة الثاذة غير الصفرية ك (Non-zero singular point ) نغرض أنّ:

$$
\begin{aligned}
& X_{t}=X_{t-1}=\zeta \\
& \zeta=\beta_{1} \zeta+\beta_{2} \zeta^{2} \\
& 1=\beta_{1}+\beta_{2} \zeta \\
& 1-\beta_{1}=\beta_{2} \zeta \\
& \zeta=\frac{1-\beta_{1}}{\beta_{2}}
\end{aligned}
$$

2- نختبر استقرارية النقطة ك كالنسبة للنموذج (21): لتكن X : $X_{t}=\zeta+\zeta_{t}$

$$
X_{t-1}=\zeta+\zeta_{t-1}
$$

نعوض عن X $X_{t}$ و $X_{t-1}$ بما يساويها في النموذج (21) بعد اعتبار تأثير Z معدوماً حيث تم اعتماده في الجزء $\zeta+\zeta_{t}=\beta_{1}\left(\zeta+\zeta_{t-1}\right)+\beta_{2}\left(\zeta+\zeta_{t-1}\right)^{2}$ الأول ومن النموذج العام فنحصل على:

$\zeta+\zeta_{t}=\beta_{1} \zeta+\beta_{1} \zeta_{t-1}+\beta_{2} \zeta^{2}+2 \beta_{2} \zeta \zeta_{t-1}+\beta_{2} \zeta_{t-1}^{2}$

$\zeta+\zeta_{t}=\beta_{1} \zeta+\beta_{1} \zeta_{t-1}+\beta_{2} \zeta^{2}+2 \beta_{2} \zeta \zeta_{t-1}$

$\frac{1-\beta_{1}}{\beta_{2}}+\zeta_{t}=\beta_{1}\left(\frac{1-\beta_{1}}{\beta_{2}}\right)+\beta_{1} \zeta_{t-1}+\beta_{2}\left(\frac{1-\beta_{1}}{\beta_{2}}\right)^{2}+2 \beta_{2}\left(\frac{1-\beta_{1}}{\beta_{2}}\right) \zeta_{t-1}$

$\frac{1-\beta_{1}}{\beta_{2}}+\zeta_{t}=\frac{\beta_{1}-\beta_{1}^{2}}{\beta_{2}}+\beta_{1} \zeta_{t-1}+\frac{1-2 \beta_{1}+\beta_{1}^{2}}{\beta_{2}}+2 \zeta_{t-1}-2 \beta_{1} \zeta_{t-1}$

$\zeta_{t}=\frac{\beta_{1}-\beta_{1}^{2}}{\beta_{2}}+\frac{1-2 \beta_{1}+\beta_{1}^{2}}{\beta_{2}}-\frac{1-\beta_{1}}{\beta_{2}}+\beta_{1} \zeta_{t-1}+2\left(1-\beta_{1}\right) \zeta_{t-1}$

$\zeta_{t}=\left(\frac{\beta_{1}-\beta_{1}^{2}+1-2 \beta_{1}+\beta_{1}^{2}-1+\beta_{1}}{\beta_{2}}\right)+\left(\beta_{1}+2-2 \beta_{1}\right) \zeta_{t-1}$

$\zeta_{t}=\left(2-\beta_{1}\right) \zeta_{t-1}$ 
النموذج الناتج يمثل نموذج انحدار ذاتي من الرتبة الأولى AR(1 بدون إزعاج ابيض (white Noise) ويكون هذا النموذج مستقراً إذا وفقط إذا ندود أن $\left|2-\beta_{1}\right|<1$

. النقاط المنفردة غير الصفرية للنموذج (21) تكون مستقرة إذا وفقط إذا

$1<\beta_{1}<3$

$X_{t}=\alpha \cos (2 \pi w t+\phi)+Z_{t}$

وبما أنّ الجزء الأول وهو النموذج

قد تم برهانه مسبقا بأنه نموذج مستقر وان النموذج المعطى هو مكون من جزأين وان الجزأين مستقرين لذلك يكون النموذج المختلط نموذجاً مستقراً بالشرط أعلاه.

12-3 الاستقرارية حسب طريقة أوزاكي: [12]

لقد تم اقتراح طريقة التقريب الخطية المحلية من قبل الباحث أوزاكي [12] استقرارية النماذج غير الخطية وتتلخص الطريقة بمرحلتين : المرحلة الأولى إيجاد النقاط المنفردة غير الصفرية للنموذج غير الخطي والمرحلة الثانية اختبار استقرارية تلك النقطة باستخدام تقنية التقريب الخطية ـ ويمكن توضيح الطريقة من خلال المثال الآتي:

مثال: [12] ليكن لدينا نموذج الانحدار الآسي الأتي: $X_{t}=\left(\phi_{1}+\pi_{1} e^{-X_{t-1}^{2}}\right) X_{t-1}+\left(\phi_{2}+\pi_{2} e^{-X_{t-1}^{2}}\right) X_{t-2}+Z_{t}$

وقد حاول الباحث (Ozaki) وضع قيود معينة على $X_{t-1}$ لغرض إيجاد النقطة المنفردة وكما يلي:

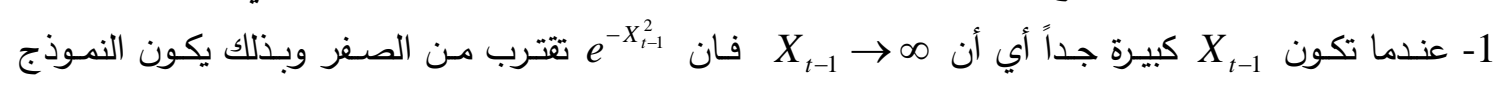
$X_{t}=\phi_{1} X_{t-1}+\phi_{2} X_{t-2}+Z_{t}$ بالثكل الآتي:

وهو نموذج انحدار ذاتي خطي من الرتبة الثانية. فتكون المعادلة المميزة بالثكل $\lambda^{2}-\phi_{1} \lambda-\phi_{2}=0$ 2-2 عندما 0 (28) $\lambda^{2}-\left(\phi_{1}+\pi_{1}\right) \lambda-\left(\phi_{2}+\pi_{2}\right)=0$

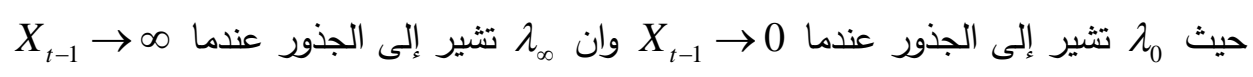
نفرض أن المعادلة (النموذج) يقترب من نقطة معينة $\xi$ إذ أن شرط وجود النقطة المنفردة هو أن تكون $0<\left(1-\sum_{i=1}^{2} \phi_{i}\right) / \sum_{i=1}^{2} \pi_{i}<1$

$$
\xi= \pm \sqrt{-\ln \left\{\left(1-\sum_{i=1}^{2} \phi_{i}\right) / \sum_{i=1}^{2} \pi_{i}\right\}}
$$

بالتالي فان قيمة $\xi$ تحسب من العلاقة الآتية: 
وان $\xi$ هي النقطة المنفردة. وبعد أن نحدد النقطة المنفردة نحاول اختبار فيما إذا كانت النقطة المنفردة مستقرة أم لا وذلك بإتباع الخطوات الآتية:

لتكن و و

$\xi_{t}=h_{1} \xi_{t-1}+h_{2} \xi_{t-2}$

$h_{1}=\left(\pi_{1}+\phi_{1} \pi_{2}-\phi_{2} \pi_{1}\right) / \sum_{i=1}^{2} \pi_{i}-2\left(1-\sum_{i=1}^{2} \phi_{i}\right) \ln \left\{\left(1-\sum_{i=1}^{2} \phi_{i}\right) / \sum_{i=1}^{2} \pi_{i}\right\}$

$h_{2}=\left(\pi_{2}+\phi_{2} \pi_{1}-\phi_{2} \pi_{2}\right) / \sum_{i=1}^{2} \pi_{i}$

النقطة المنفردة تكون مستقرة إذا وفقط إذا كانت جذور المعادلة المميزة

$\lambda^{2}-h_{1} \lambda-h_{2}=0$

تقع داخل دائرة الوحدة، لاحظ ( Ozaki (12) م (12) ـ

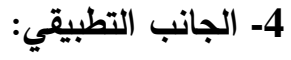

تم في هذا البحث دراسة السلسلة الزمنية للمعدلات الشهرية لدرجات الحرارة في مدينة بيجي ( شكل- 1). وتم إيجاد نماذج مختلفة لهذه السلسلة وبالاعتماد على المراحل الأساسية الثلاثة لطريقة (Box-Jenkins) في التحليل

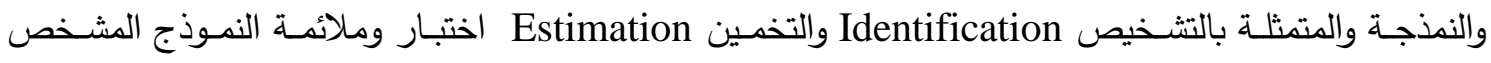
تمن Diagnostic checking. (لاحظ [4]) وقد تم بناء نماذج مختلفة للسلسلة أعلاه حيث لوحظ أن السلسلة دوريـه تعيد نفسها كل (12) شهر تقريباً. ولزيادة في التفاصيل لاحظ [1] والنماذج التي حصلنا عليها كالآتي: 1-4 النموذج التوافقي مضافاً له حد الخطأ: بالاعتماد على البرنامج الجاهز Statistica تم نمذجة السلسلة بالنموذج التوافقي الآتي: $X_{t}=-14.7254 \cos (0.08333 * t)-2.19991 \sin (0.08333 * t)+Z_{t}$

$\sigma_{z}^{2}=227.1697$

$\mathrm{NBIC}=5.458398$

إذ أن NBIC=

2-4 نموذج الطيف المختلط الخطي:

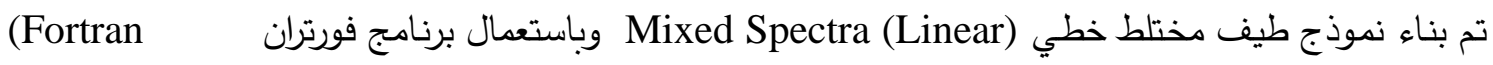

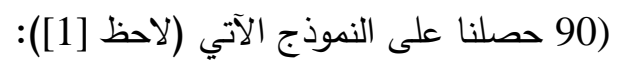

$X_{t}=-12.7254 \cos (0.08333 * t)-2.19991 \sin (0.08333 * t)+0.0595 X_{t-1}+Z_{t}$

$\sigma_{z}^{2}=179.8894$

$\mathrm{NBIC}=5.209079$ 


\section{3-4 نموذج الطيف المختلط غير الخطي:}

على الرغم من أن تباين البواقي لنموذج الطيف المختلط الخطي اقل من تباين البواقي للنموذج التوافقي لكن

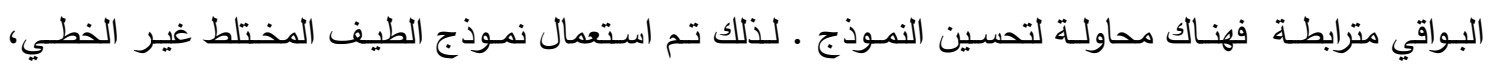
وباستعمال برنامج فورتران (Fortran 90) • حصلنا على النموذج الآتي لاحظ (ملحق (1)) $X_{t}=-12.7254 \cos (0.08333 * t)-2.19991 \sin (0.08333 * t)+$

$$
0.10747 X_{t-1}+0.02178 X_{t-1}^{2}+Z_{t}
$$

$$
=99.38671 \sigma_{z}^{2}
$$

$\mathrm{NBIC}=4.615749$

\section{4-4 نموذج الانحدار الذاتي والأوساط المتحركة المندمج (المضاعف) الموسمي}

\section{SARIMA $(1,0,0)(2,0,0) S$}

بالعودة إلى الوصف البياني للسلسلة وبعد اخذ اللوغارتم الطبيعي للبيانات والفرق الموسمي (12) لان السلسلة تعيد

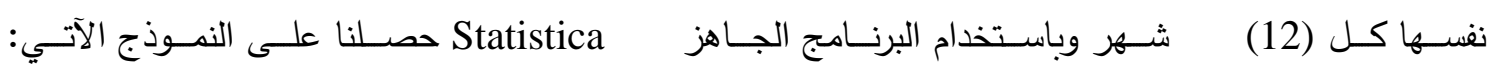

$$
X_{t}=\phi_{1} X_{t-1}+\phi_{1 s} X_{t-1}+\phi_{2 s} X_{t-1}+Z_{t} \quad \text { والذي يكتب بالثكل: SARIMA(1,0,0)(2,0,0)s }
$$

والجدول الأتي يمثل النماذج التي حصلنا عليها مع الصفات الإحصائية لها. جدول رقم (1). جدول مقارنة بين النماذج المقترحة

\begin{tabular}{|c|c|c|c|c|}
\hline stat & NBIC & rss & Corr. \% & mean \\
\hline Harmonic Model & 5.458398 & 227.1697 & $80 \%$ & 28.60514 \\
\hline $\begin{array}{c}\text { Linear mixed } \\
\text { spectral model }\end{array}$ & 5.209079 & 179.8894 & $80 \%$ & 25.34633 \\
\hline $\begin{array}{c}\text { Non-Linear mixed } \\
\text { spectral model }\end{array}$ & 4.615749 & 99.38671 & $80 \%$ & 2.682353 \\
\hline $\begin{array}{c}\text { SARIMA } \\
(1,0,0)(2,0,0)_{\mathrm{s}}\end{array}$ & -3.1048 & 0.042688 & $0 \%$ & 0.01959 \\
\hline
\end{tabular}

ومن الجدول رقم (1) نلاحظ أنّ بواقي النمـاذج مترابطة بشكل كبير والتباين (rss) والوسط للبواقي كبير أيضـا باستثناء النموذج الموسمي الذي تكون بواقيه غير مترابطة وتباين البواقي والوسط قريب جداً من الصفر مما يشير تفوّق النموذج الموسمي 12 SARIMA (1,0,0)(2,0,0) على بعض النماذج. لذلك يمكن استخدامه لتمثيل السلسلة على الرغم من أن بواقيه لا تتبع التوزيع الطبيعي.

Forecasting 5-4

سنحاول التتبؤ بالقيم المستقبلية للمعدلات الثهرية لدرجات الحرارة العظمى ولفترة (12) شهراً. وباستعمال طريقة بوكس - جنكز في التتبؤ وباستعمال البرنامج الجاهز Statistica الذي يستخدم طريقة التحويل المباشرة في التتبؤ 


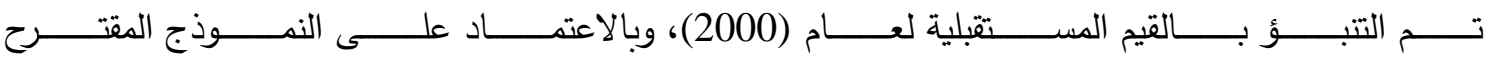
SARIMA(1,0,0)(2,0,0) والجدول الآتي جدول رقم (2) يمثل مقارنة بين القيم المتنبأ بها والقيم الحقيقية. الجدول رقم (2). جدول مقارنة بين القيم المتتبأ بها من النموذج المقترح والقيم الحقيقية

\begin{tabular}{|c|c|c|}
\hline شهراً 12 & $ت$ \\
\hline القيمة المتكهن بها لمدة القيمة الحقيقية 12 شهرا & 13.5 & 1 \\
\hline 14.7505 & 18.5 & 3 \\
\hline 16.8046 & 20.6 & 4 \\
\hline 19.3804 & 29.7 & 5 \\
\hline 23.8972 & 35.1 & 6 \\
\hline 27.8980 & 40.8 & 7 \\
\hline 31.8662 & 46.6 & 8 \\
\hline 32.7701 & 44.4 & 9 \\
\hline 32.8978 & 38.2 & 10 \\
\hline 28.6208 & 30.2 & 11 \\
\hline 25.3720 & 22.9 & 12 \\
\hline 20.2003 & 15.8 & \\
\hline 16.4487 & &
\end{tabular}

من الجدول نلاحظ أن القيم المتنبأ بها لفترة 12 شهراً من النموذج الموسمي المضاعف هي قريبه نوعاً ما من القيم الحقيقية وذات صفات دوريـة مما يشير إلى كفاءة النموذج المقترح والذي يمثل النموذج الملائم لسلسلة المعدلات الثهرية لدرجات الحرارة العظمى لمدينة بيجي. 6-4 استقرارية النماذج المدروسة: 1-6-4 النموذج التوافقي مضافاً له حد الخطأ:

من ملاحظة الفقرة (4-1) وجدنا أن القيمة المتوقعة والتباين والتغاير كميات ثابتة لا تعتمد على الزمن . مما يثير إلى أن النموذج التوافقي مستقر وفي التطبيق:

$$
\begin{aligned}
& f\left(\phi_{j}\right)=\frac{1}{2 \pi}, j=1,2,3, \ldots \ldots . ., m \\
& \alpha_{j}=\sqrt{A_{j}^{2}+B_{j}^{2}}=\sqrt{(-14.7254)^{2}+(-2.19991)^{2}}=\sqrt{221.677} \\
& \gamma_{0}=\sum_{j=1}^{m} \frac{\alpha_{j}^{2}}{2}+\sigma_{z}^{2}=\sum_{j=1}^{m} \frac{(\sqrt{221.677})^{2}}{2}+\sigma_{z}^{2}=110.8385+227.1697=338.0082 \\
& \gamma_{r}=\sum_{j=1}^{m} \frac{\alpha_{j}^{2}}{2}\left[\cos \left(W_{j} r\right)\right]=\sum_{j=1}^{m} \frac{(\sqrt{221.677})^{2}}{2}\left[\cos \left(0.08333^{*} r\right)\right] \\
& =\sum_{j=1}^{m} 110.8385^{*}\left[\cos \left(0.08333^{*} r\right)\right]
\end{aligned}
$$




$$
\begin{aligned}
\rho_{r} & =\frac{\gamma_{r}}{\gamma_{0}}=\frac{\sum_{j=1}^{m} \alpha_{j}^{2}\left[\cos \left(W_{j} r\right)\right]}{\sum_{j=1}^{m} \alpha_{j}^{2}+2 \sigma_{z}^{2}}=\frac{\sum_{j=1}^{m}(\sqrt{221.677})^{2}[\cos (0.08333 * r)]}{(\sqrt{221.677})^{2}+2 * 227.1697} \\
& =\frac{221.677 * \cos (0.08333 * r)}{676.0164}=0.327917 * \cos (0.08333 * r)
\end{aligned}
$$

2-6-4 نموذج الطيف المختلط الخطي:

لاحظنا أنّ نموذج الطيف المختلط الخطي من الفقرة ( 4-2) مستقر لان العزوم لا يعتمد على t ، وفي التطبيق حصلنا على:

1) $f\left(\phi_{j}\right)=\frac{1}{2 \pi}$

$$
\begin{aligned}
& \alpha=\sqrt{\alpha_{1}+\alpha_{2}}=\sqrt{(-12.7254)^{2}+(-2.19991)^{2}}=\sqrt{221.677} \\
& \text { 2) } \gamma_{0}=\frac{\alpha^{2}+2 \sigma_{z}^{2}}{2\left(1-\beta^{2}\right)}=\frac{(\sqrt{221.677})^{2}+2 * 179.8894}{2\left(1-(0.0595)^{2}\right)}=\frac{616.6704}{1.99292}=309.4307 \\
& \gamma_{k}=\frac{\alpha^{2}(\cos (0.08333 * k)}{2\left(1-\beta^{2}\right)}
\end{aligned}
$$

3) $\gamma_{k}=\frac{221.677 *(\cos (2 \pi w k)}{1.99292}=111.2323 *(\cos (0.08333 * 2 \pi k))$

4) $\rho_{k}=\frac{\alpha^{2}(\cos (2 \pi w k))}{\alpha^{2}+2 \sigma_{z}^{2}}=\frac{221.677 *(\cos (0.08333 * k))}{221.677+2 * 197.4967}$

$$
=0.359474 *(\cos (0.08333 * k))
$$

نلاحظ أن العزوم لا تعتمد على t مما يشير أن النموذج مستقر. 3-6-4 نموذج الطيف المختلط غير الخطي:

$\xi=\frac{1-\beta_{1}}{\beta_{2}}$

بما أنّ النقطة الثابتة التي تم إيجادها لاحظ الفقرة (4-3) $1<\beta_{1}<3$

ويكون هذا النموذج مستقرا إذا وفقط إذا

$$
1<\beta_{1}<3
$$$$
X_{t}=\alpha \cos (2 \pi w t+\phi)+\beta_{1} X_{t-1}+\beta_{2} X_{t-1}^{2}+Z_{t}
$$

نموذج الطيف المختلط غير الخطي يمكن التعبير عنه بالثكل

$$
X_{t}=\mu+\alpha \cos (2 \pi w t+\phi)+\beta_{1} X_{t-1}+\beta_{2} X_{t-1}^{2}+Z_{t}
$$

ويمكن أن يعبر عنه بالثكل

$$
\text { والنموذج الذي حصلنا عليه لتمثيل سلسلة المعدلات الثهرية لدرجات الحرارة لمدينة بيجي هو }
$$
$X_{t}=-12.7254 \cos (0.08333 * t)-2.19991 \sin (0.08333 * t)+$

$$
0.10747 X_{t-1}+0.02178 X_{t-1}^{2}+Z_{t}
$$




$$
\text { أي أن }
$$
وعليه تكون النقطة المنفردة هي

$\xi==\frac{1-0.107}{0.02178}=41$

وعند اختبار استقرارية النقطة المنفردة وملاحظة المعادلة (22) حيث أن شرط الاستقرارية هو $1<\beta_{1}<3$

ومن النموذج أعلاه نجد أن 4-6-4

النمـوذج المقترح هـ 12 SARIMA بعد اخذ اللوغـارتم الطبيعسي للبيانـات الأصـلية أي أن

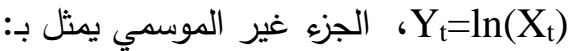

$\phi_{p}(B)=1-\phi_{1} B$

$\phi_{p s}(B)=1-\phi_{1 s} B^{s}-\phi_{2 s} B^{2 s}$

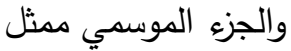
وان

$\phi_{1}=0.9797$

$\phi_{1 s}=0.4274$

$\phi_{2 s}=0.3422$

يكون النموذج مستقرا إذا كانت جذور المعادلة المميزة لنموذج الانحدار غير الموسمي من الرتبة الأولى تقع داخل دائرة الوحدة. أي أن شرط الاستقرارية هو 1

$\phi_{p}(B)=0$ وباستخدام المعادلة المميزة للجزء غير الموسي:

$\lambda-0.9797=0$

$\lambda=0.9797$

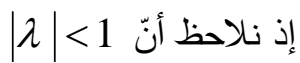

مما يثير إلى أن الجزء غير الموسمي مستقر وبما أن معالم نموذج الانحدار الذاتي الموسمي 12 (2,0,0) هي $\phi_{1 s}=0.4274$

$\phi_{2 s}=0.3422$

$\lambda^{2}-0.4274 \lambda-0.3422=0$

$\lambda_{1}=-0.2137-0.5446 \mathrm{i}$

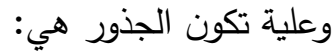

$\lambda_{2}=-0.2137+0.5446 \mathrm{i}$

$\left|\lambda_{1}\right|=0.585$

$\left|\lambda_{2}\right|=0.585$ 
وبما أن جذور المعادلة المميزة للجزء الموسمي وكذلك جذور المعادلة المميزة للجزء غير الموسمي تقع داخل دائرة

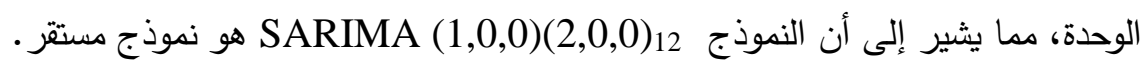

5- الاستنتاجات والتوصيات

من خلال هذه الدراسة توصلنا إلى الاستتتاجات الآتية: 1- النماذج التوافقية هي نماذج مستثرة.

2- إنّ نموذج الطيف المختلط الخطي نموذج مستقر أيضا باعتباره حاصل جمع نموذجين مستقلين نموذج توافقي ونموذج خطي.

3- نموذج الطيف المختلط غير الخطي هو حاصل جمع نموذجين (توافقي+ نموذج غير خطي) وقد وجدنا أن

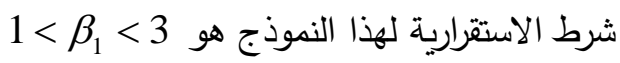

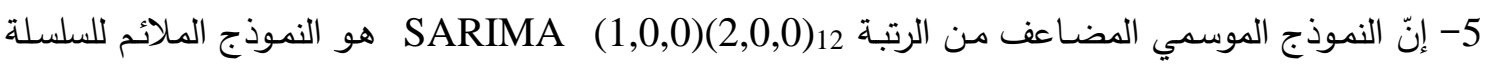

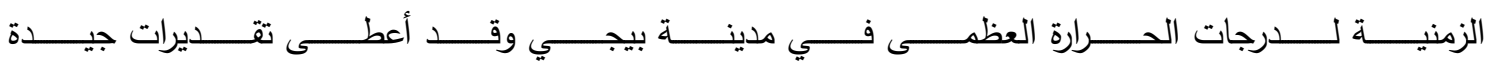
(للتبوء بالقيم المستقبلية) وقريبة من قيم الواقع الفعلي. ونوصي بإجراء دراسة متكاملة لنماذج طيف مختلط غير خطي، بحيث يكون الجزء غير الخطي هو نموذج أسي أو أو نموذج عتبة وبرتب عليا. 


$$
\begin{aligned}
& \text { المصادر } \\
& \text { الحمداني، رعد عواد حميد (2008) "استقرارية بعض نمـاذج السلاسل الزمنيـة مـع تطبيقاتها " رسالة } \\
& \text { ماجستير غير منشورة، قسم الرياضيات كلية التربية، جامعة تكريت. } \\
& \text { الحيالي، أزهر عباس محمد (2005) "استقرارية بعض نمـاذج الانحدار الذاتي اللاخطيـة " أطروحسة } \\
& \text { دكتوراه غير منشورة مقدمة إلى كلية التربية، جامعة تكريت. } \\
& \text { العبيدي، عبد الغفور جاسم، (1989) " تحليل ونمذجة السلسلة الزمنية لدرجات الحرارة في مدينة الموصل } \\
& \text { " رسالة ماجستير غير منشورة، قسم الرياضيات، كلية العلوم، جامعة الموصل. } \\
& \text { العبيدي، عبد الغفور جاسـ، وفـاء محي الدين (2003) " نــوذج السلسـلة الزمنيـة للمعدلات الثـهرية }
\end{aligned}
$$

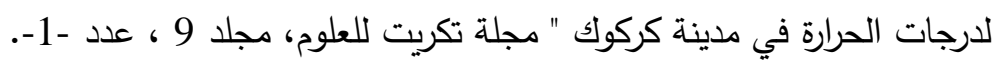

$$
\begin{aligned}
& \text { المهداوي، هيفاء جعفر، (1991) "الانحدار الذاتي غير المستقر ذو الدرجات الدنيا "، أطروحة دكتوراه } \\
& \text { غير منشورة، كلية الإدارة والاقتصاد، الجامعة المستتصرية. }
\end{aligned}
$$

[6] Bloomfield, P. (1976), "Fourier Analysis of Time series: An Introduction. John Wiley and son Inc. New York.

[7] Chatfield (1978) "The analysis of time series: Theory Practice" Chapman and Hall, London .

[8] Chatfield, Christopher.(1984) "The analysis of time series : 4th Edition Chapman and Hall.

[9] FriedricK, I. and Kurt Hornik, (1999) "stationarity and stability of Autoregressive Neural Network processes". MIT Prss,Cambridge, MA,USA

[10] Giovanni Fonseca (2004) "On the Stationarity of first order non linear \& time series Models: Some Developments" studies in Non linear Dynamics Econometrics: Vol 8: No. 2, Article 12.

[11] Ozaki,T.(1982) "Non linear time series stochastic processes and Dynamical system" Handbook of statistics, Vol.5,Itd.

[12] Ozaki, T.(1985) "Non linear time series models and Dynamical system" E.J. Hannan, P.R. Krishnaiah, M, M.Rao, eds., Handbook of statistics, Vol.5, pp2583.

[13] Priestley, M.B.(1982) "Spectral Analysis and time series", Academic press, Inc., London.

[14] Priestly, M.B.(1988) "Non linear and stationary time series analysis": Academic. Press London.

[15] Tong .H. (1990) "Non linear Time series; A Dynamical system Approach". Oxford University Press, London. 


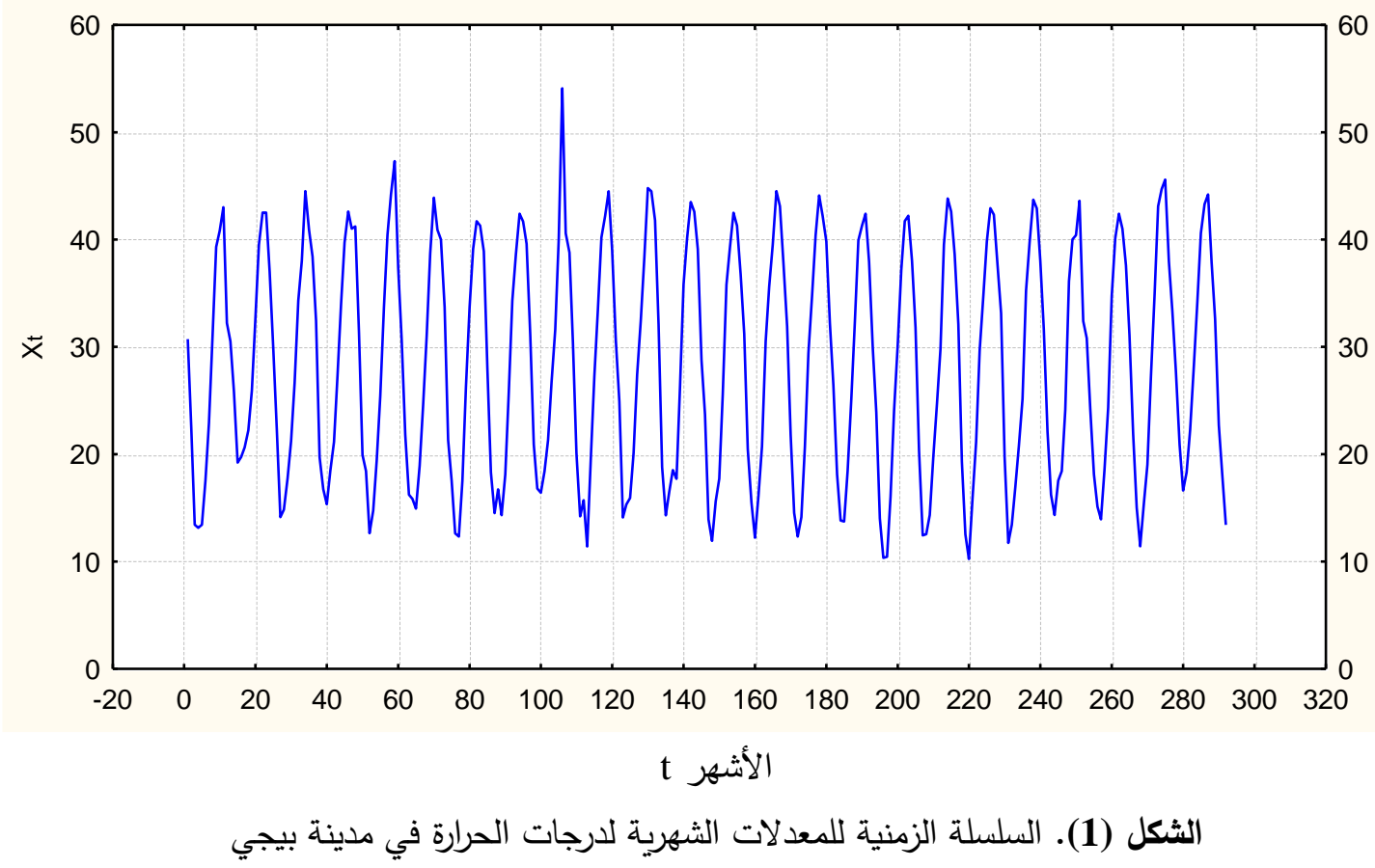

